and of the Bureau des Longitudes, and was for some time a member of the Council of the Observatory. It is understood that M. Bardoux suggested the adoption of the system in operation at the United States Naval Observatory at Washington, and to model the great Paris Observatory after that institution; and as stated above, the appointment of Admiral Mouchez as director, and $M$. Lœwy as sub-director, are at present intended to be limited to five years, with the same restriction as to future nominations.

M. Maurice Lœewy, who was born at Pesth in 1834 , commenced his astronomical career at the Imperial Observatory of Vienna, under the late Prof. Carl von Littrow, on whose recommendation he was transferred to the Observatory of Paris by Leverrier, in 1860 . At Vienna he was much occupied with the calculation of the orbits of comets, including the great comet of Donati in I 858 , for which body he was one of the first to establish elliptical elements. He succeeded Laugier, as one of the astronomers of the Bureau des Longitudes in 1872, and since 1874 has been charged with the preparation of the Connaissance des Temps, the French national ephemeris, and the Anmuaire, works which have greatly benefited by his energetic superintendence. Under Delaunay's rule, M. Lœwy occupied the position of subdirector of the Observatory of Paris, charging himself with the meridian observations.

The installation of Admiral Mouchez took place on Saturday by the Council of the Observatory, of which M. Dumas is president.

\section{PROF. W. M. GABB}

WE greatly regret to hear of the death from consumption, on May 30, at his residence in Phila delphia, of Prof. William M. Gabb, who for many years has occupied a very prominent place among American naturalists.

He was born on January 20, I 839 , in Philadelphia, and was educated at its High School, being one of the many graduates of whom that institution had reason to be proud. As a boy he was especially interested in mineralogy and palreontology, and at an early age was so fortunate as to secure an engagement with Prof. James Hall, where he had ample opportunity of indulging his tastes. Returning to Philadelphia, he became a member of the Academy of Natural Sciences, and soon commenced the critical study of the fossil invertebrates of the United States, especially those of the cretaceous formation.

In 1860 he entered the service of the Geological Survey of California, under Prof. J. D. Whitney, but returned to the East in 1868 , and undertook the geological survey of their lands for the Santo Domingo Land and Mining Company, which, however, was made to cover a considerable portion of the Dominican Republic, and to which he subsequently made several successive visits for the purpose of continuing his work.

During his connection with the Geological Survey of California he made an extended exploration of the peninsula of Lower California, collecting much important geological and biological material.

In 1873 he became connected with the Costa Rican Government, undertaking a general geological and topographical survey of its territory, and combined with it very extensive researches into its natural history and ethnology, sending bis collections to the National Museum in Washington. This labour occupied him for about three years. The results of his work have been given to the public in various forms. A full account of the topography, with an elaborate map, appeared in Petermann's Mittheilungen, and a paper on the ethnology of the native tribes, published by the American Philosophical Society, is one of standard value.
In the autumn of 1876 he revisited San Domingo, returning to the United States in March last. For many years he has been threatened with pulmonary disease, the extension of which has been checked by his abode in subtropical regions. The unfavourable symptoms, however, increased of late, and he succumbed shortly after his return to Philadelphia.

Dr. Gabb left an extensive manuscript on the geology and palæontology of Costa Rica, which will be published ere long under competent supervision, thus closing a career of energy and activity, not only in the prosecution of researches, but in the elaboration of their results, which has been seldom equalled by a man of his age. It is very rare, indeed, that one man has accomplished so much in so many distinct branches-in geology; 'geography, palaontology, ethnology, \&c.--as: the subject of our present notice.

ON THE ANATOMY OF THE ORGAN OF HEARING IN RELATION TO THE DISCOVERY OF THE PRINCIPLE OF THE MICROPHONE OF PROF. D. E. HUGHES, AND THE MAGNOPHONE OF MR. W. L. SCOTT, A.S.T.E. ${ }^{1}$

THE two gentlemen whose names appear in the heading of this paper seem to have arrived at the same important result, viz., the extraordinary effect of mobile particles in transmitting sound under certain conditions, by quite independent research. In perusing the interesting accounts of the microphone in several scientific journals, but especially an article in the Electrician for May 25, in which number also will be found Mr. Scott's statement of the principle, it occurred to me that the transmitting power of the otoconia and otoliths in the ears of animals bore very pertinently upon this question. We find otoconia, or numerous minute particles in all the Vertebrata, with perhaps the exception of the bony fishes which have single concretions, or the union of many. in one. Otoconia are also found in the Tetrabranchiate Cephalopoda (Nautilus, Fig. I), the whole of the Pteropoda, in the Pumonifera inoperculata, or rather the bisexual Pulmonifera (snails and slugs, Fig. 3), there being an operculum in Amphibola. On the other hand, in the Dibranchiate Cephalopoda (Sepia, Fig. 2), all the Heteropoda (Fig. 5) and the unisexual operculate Pulnonifera (Fig. 4) the ear-sacs contain single otoliths.

It will be thus seen that the nature of the auditory concretions is by no means an unimportant element in the classification of animals. ${ }^{2}$ Prof. Huxley alludes to the genus Polyophthalmus, an Annelidan with eyes in every segment, as a remarkable fact, but this is excelled by his notice of otoliths in the tail of Mysis fexuosa, ${ }^{3}$ a little pelagic crustacean which I have often had the opportunity of examining.

Every physiologist is aware that there are structural particulars in the ears of Vertebrata which show clearly that nature's philosophy is of a more profound character than that to which man has hitherto attained. Indeed if we study the simplest ears in creation, those, for example, of the common Snail and of the Pcriwinkle, a most interesting problem is presented to us to solve, namely, the precise function of the numerous otoconia in one case, and of the single otoliths in the other. It is commonly granted that these concretions augment the sonorous undulations by resonance, a view which is borne out by several considerations. If we take two stones and strike them together under water, the head also being immersed, the collision will produce a very loud and peculiar sound, but in order to make the minute otoconia impress one another

By John Denis Macdonald, M.D., F.R.S., Dep. Ins. Gen. R.N., \&zc.

See which a classification of the Gasteropoda has been attempted.

3 See $A$ nn. and Mag. of Nat. Hist. for May, x851. 
with the rhythmical flow of the undulations of sound, they must be poised off from the walls of the auditory sac. And this is effected, in some instances, by little tubercles on the inner surface as in the unttle fish or as in the Heteropod, as shown in Fig. $5(h)$, the auditory organ of Cerophora, in which also large vibratile cilia effect a continual rotatory motion of the spherical otolith. In other cases when otoconia are present, a fine ciliated lining not only prevents the contact of these minute particles with the walls of the sac, but keeps them in constant motion, jostling one another in a remarkable manner. Now when acoustic waves are passing over the auditory organ, it is easy to perceive how their impulses may be imparted to the otoconia, and thereby communicated with augmented effect to the auditory centre.

Whatever may be the intrinsic nature of nervous force it exhibits unmistakable polar properties which would

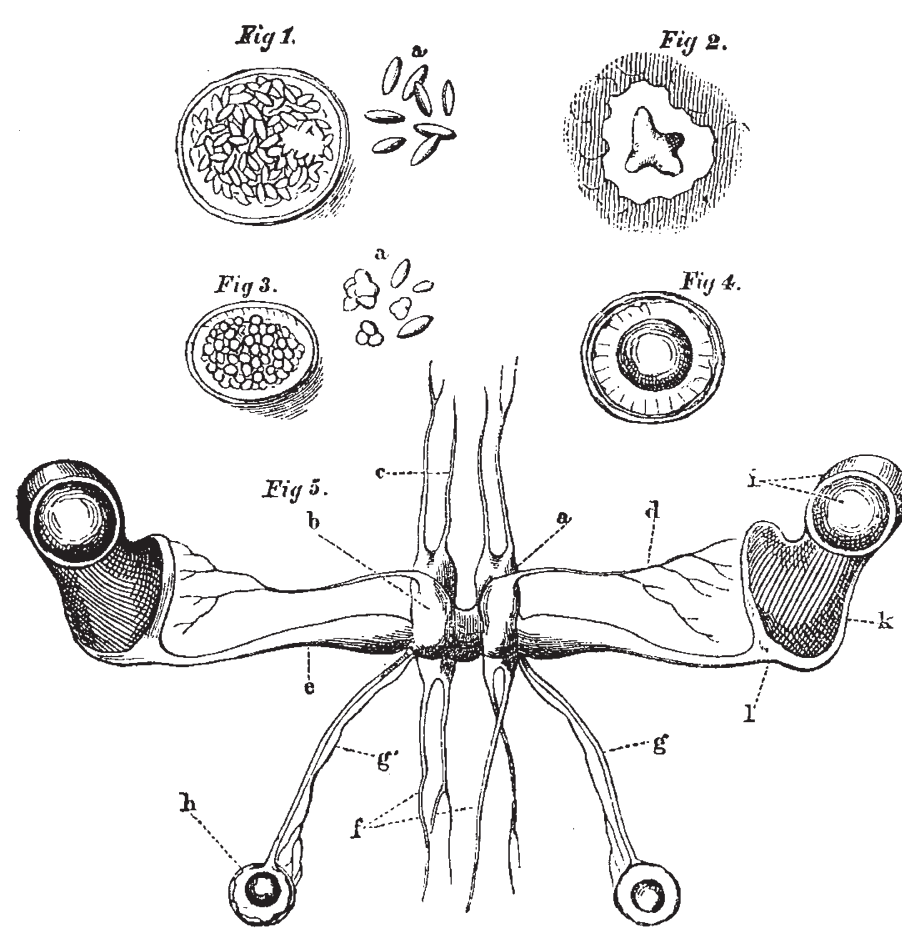

Fig. r.-Auditory sac of Nantilus. a, otoconial particles, elliptical.

FIG. 2.-Section of auditory cavity of Sepia, with a somewhat cruciform otolith.

FIG. 3-- Auditory sac of Limtax atelis, \&c. $a$, otoconial particles, some of them com pound, others of variable sixe.

Fič. 4- - Auditory sac of Litorina, Ceomelania, or Cyclostomo.

IG. 5.-Cerebrold ganglia and organs of vision and hearing in Cerophora. a, upper

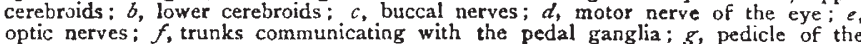
optic nerves: $f$, trunks communicating with the pedal ganglia; $g$, pedicle of the auditory sac; $g$, nerve distributed to the auditory sac; $h$, auditory sac with
otolith $i$, meniscus, and lens of the eye $: k$, body of the eye; retina.

thus place it in the category of the electric force, though no one now would attempt to reason out the identity of the two. It is, however, a new idea that nervous force in the function of auctition plays an analogous part to the electric force in the case of the microphone. Moreover, if we look to the anatomy of the ear, we see that provision for a complete circuit is made. Take, for example, the auditory sac $(h)$, pedicle $(g)$, and nerve $\left(g^{\prime}\right)$ of Cerophora (Fig. 5). Nothing would appear to be wanting if we admit the nervous centre to be the equivalent of the battery, From all this it would seem to be a rational hypothesis that nervous force in traversing the circuit just indicated wnder the influence of sonorous undulations is actually transmitted from particle to particle of otoconia, or through the revolving otolith, as the case may be. But while the microphone, or the principle involved in it, affords us some additional light in relation to the physiology of hearing, the study of the anatomy of the ear might give electricians some valuable hints as to the construction of transmitting apparatus. One of Prof. Hughes' transmitters so exactly resembles the natural arrangement of the par the higher animals, that some ew remarks on this subject may not be out of place here. Many years ago (1847-48) I noticed that a small piece of steel casually lying in the box of a square pianoforte reproduced, with great fidelity, any note, or number of notes, touched on the instrument, by the impact of its own weight meeting the vibrations of the sound-board beneath. Here was, in effect, the basis of the telephone, and I would indeed have anticipated the wisdom of the age had I known bow to call in the aid of electricity in the simple way that this has been done by Prof. Bell. The fact, however, was made the subject of a paper published in the Medical Gazette, in which the Membrana tympani and the Malleus were compared with the soundboard and steel rod in the case referred to. Moreover, the inference was drawn that while the membrane communicates its vibrations to the ossicles, or small bones, it also causes the malleus to percuss the face of the incus responsively to the rapid and varied impressions made upon it, a view which borrows additional weight from the fact that in the frog articulation is still persistent where, from the absence of muscles for adjustment it might be considered to be quite unnecessary.

It should also be remembered the handle of the malleus extends like a radius from the centre to the circumference of the drum membrane, so as thus to include, without impeding, its three vibrating segments. The centre gives the key-note the circumference the fifth, and the intervening region the third. Only add to this the aural lens or lenticles, the otoliths, or the otoconia, with the light which the microphone has cast upon their function, and we are enabled to form a better conception of the physiology of hearing than has hitherto been possible. The accentuations, piano, forte, \&c., in musical pieces, are marked with extreme accuracy, and should the parts of the music be deranged by a defect in time, an uneasy jog will be produced in the auditory apparatus, hence the antipathy of the mind to any erratic deviation in this respect. It is very remarkable that the malleus and incus (the hammer and the anvil) should correspond not only in figure, but also in function, to the objects from which their respective names are derived, for as we have already seen, the uses of the bammer and anvil, as employed in mechanics, are literally fulfilled by the malleus and incus answering very important ends in the faculty of audition. By the action of one upon the other, sounds are not only correctly transmitted to the auditory centre, but an accurate register of time, grace, and style, is effected in the manner above explained. Thus the physical organisation itself may be shown to be the natural preceptor of the mind. ${ }^{1}$

1 Dr. F. de Chaumont writes as follows :-

Since Dr. Macdonald's paper was forwarded to you the appearance of Mr. Blyth's has added still stronger confirmation to the analogy between the microphone and the auditory apparatus of the mollusca, \&c. One point in particular is the necessity of moisture of some kind or other, as a medium between the conducting particles, shown by the fact that even the watery vapour from the breath of the experimenter produced a sensible increase in the strength of the sounds elicited; and further, that the addition of simple water to the cinders was productive of still more striking effects. All this bears out the view taken by Dr. Macdonald that we have in the otoconia, endolymph and vestibule of the ear the most complete type of a microphone. It would be well to try the free suspension of good conducting particles in a non-conducting fluid medium, sufficiently limpid to offer the least possible impediment to their movement.

The oscillation of the transmitter as a whole by mechanical means ought also to be tried in order to imitate the effect of vibratile cilia in the case of the auditory vestibule of animals.

Army Medical School, Netley, June 20 